\title{
FLEXOPHORA OPHIDII n. g., n. sp. UN DICLIDOPHORIDAE (MONOGENEA) PARASITE D'OPHIDIUM BARBATUM (L.) (TELEOSTEI)
}

\author{
Par MARIA PROST et LouIs EUZET
}

Au cours de recherches sur les Monogènes parasites des Poissons de la région de Sète, nous avons trouvé, sur les branchies d'Ophidium barbatum (L.), huit individus d'une espèce de Diclidophoridae, qui nous paraît être nouvelle pour la science. Nous en donnons ci-après une brève description :

Hôte : Ophidium barbatum (L.), Ophidiidae.

Habitat : Branchies.

Localité : Sète (Hérault).

Le corps, petit, assez trapu, mesure de $1,50 \mathrm{~mm}$. à $2,75 \mathrm{~mm}$. de long et de $0,40 \mathrm{~mm}$. à $0,85 \mathrm{~mm}$. de large.

La bouche est antérieure, ventrale, subterminale. A la partie postérieure, le hapteur se compose de huit pinces en quatre paires symétriques. Chacune de ces pinces est portée par un court pédoncule musculeux (fig. 1).

Ces pinces ne sont pas toutes de même taille. Celles de la première paire, la plus inférieure, sont légèrement plus petites.

Les pinces mesurent:

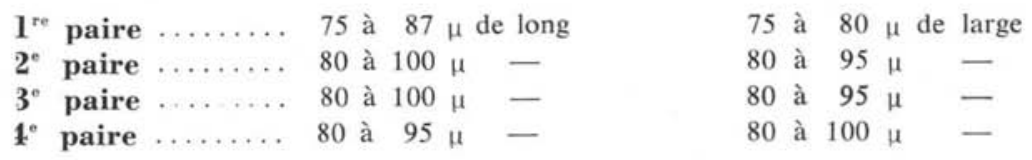

Les deux mors de chaque pince sont soutenus par des pièces rigides. Ces pinces, ressemblant à des pinces de Diclidophora, nous emploierons, pour désigner les pièces rigides ou sclérites, la nomenclature établie en 1958 par J. Llewellyn pour ce genre, nomenclature qui a été reprise en 1961 par E.-S. Robinson.

D'après cette nomenclature, la mâchoire postérieure comprend un sclérite médian, $f$, et quatre sclérites périphériques: deux du côté distal, $g^{1}$ et $g^{2}$, et deux du côté proximal, $i$ et $k$ (fig. 7). 

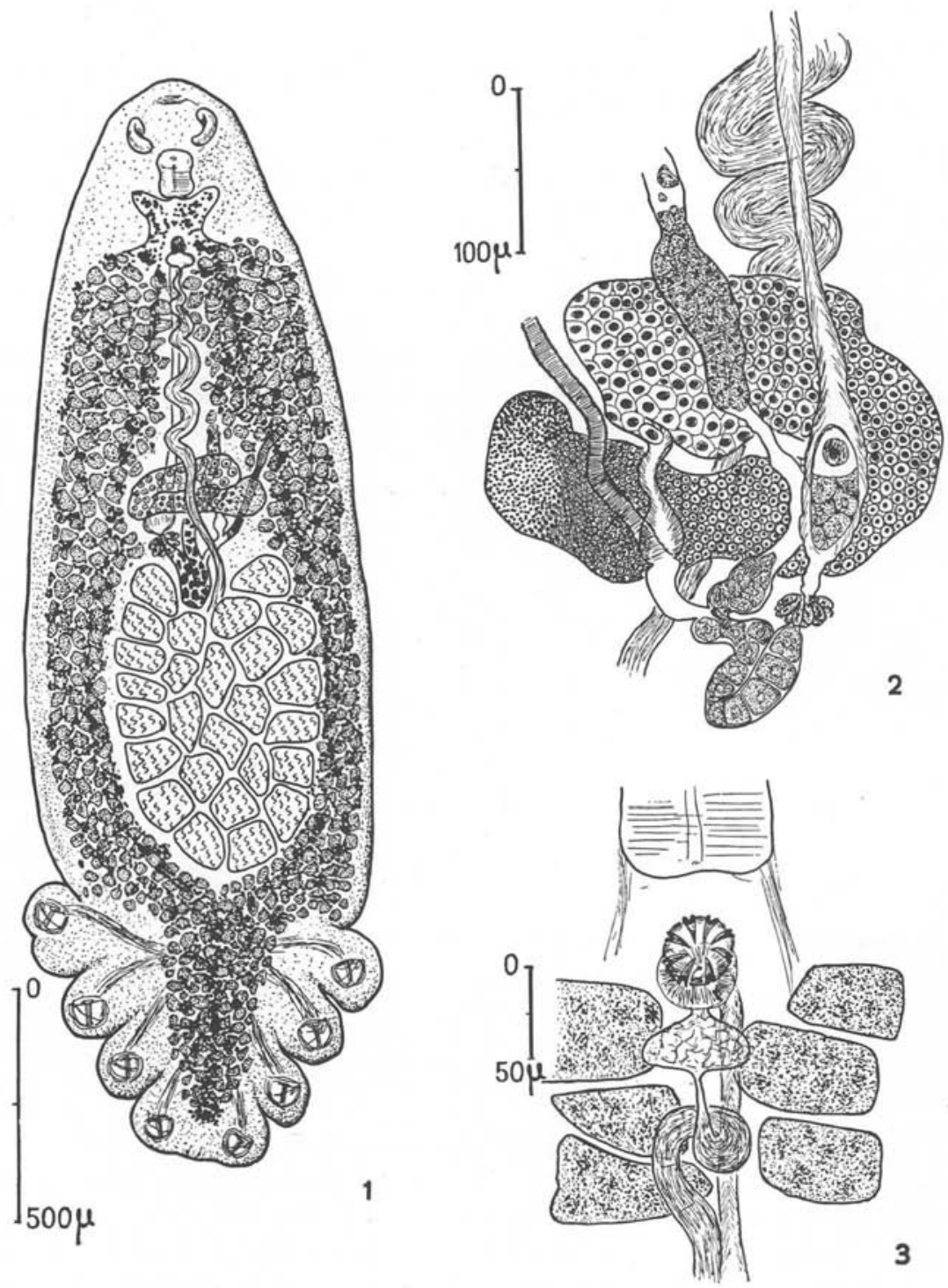

Fig. 1. - Flexophora ophidii n. g., n. sp. Vue dorsale. D’après une préparation in toto

FIG. 2. - Complexe génital, vue ventrale

Fig. 3. - Ouverture génitale et partie terminale du canal déférent

Ann. De Parasitologie, $\mathrm{T}$. XXXVII, $\mathrm{N}^{\circ} 3 .-1962$. 
Dans l'espèce qui nous intéresse, $f$ est très allongé, creusé en gouttière et élargi en $\mathbf{T}$ à chaque extrémité.

L'élargissement proximal est légèrement plus important que le distal (fig. 4).

Les pièces $g^{1}$ et $g^{2}$ sont allongées et presque contiguës au niveau de l'extrémité distale de $f$.

$i$ fait suite à $g^{1} ; k$ à $g^{2}$. Ces pièces $i$ et $k$ sont un peu plus courtes et plus trapues que $g$. En outre, $i$ forme un élargissement en crochet à son extrémité proximale.

Chez Diclidophora, il y a dans la mâchoire antérieure une pièce centrale, $a$, formant sur la moitié de la mâchoire une extension lamelleuse, $b$.

Les bords de la mâchoire sont soutenus par le sclérite $c$ du côté de $b$ et $d$ de l'autre. Ces pièces se replient en $c^{2}$ et $d^{2}$ et, passant par-dessus l'articulation de la mâchoire, donnent les pointes $c^{3}$ et $d^{3}$ (fig. 7).

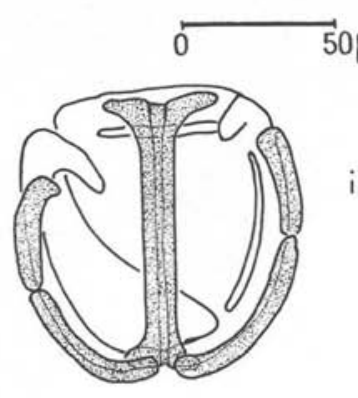

4

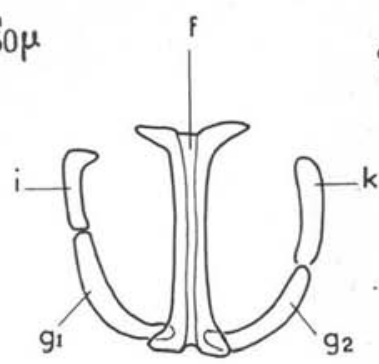

5

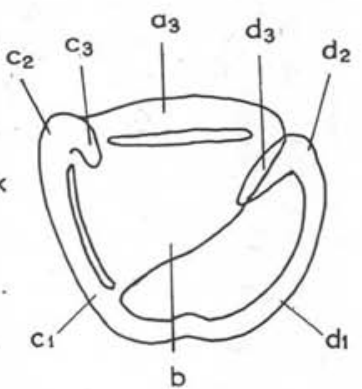

6

Disposition des selérites de la pince de Flexophora ophidii n. g., n. sp.

Fia. 4. - Pince in toto, vue postérieure

Fıg. 5. - Mâchoire postérieure isolée, vue antérieure

Fıg. 6. - Mâchoire antérieure isolée, vue antérieure (Lettres dans le texte)

Dans l'espèce étudiée, $a$ est très différent. Il est réduit à une bande transversale représentant ce que Llewellyn a nommé $a^{2}$ et $a^{3}$. Sur cette partie vient s'articuler $f$. Cette bande transversale $a^{3}$ se soude d'un côté sur $c^{3}$ très court et, de l'autre, se replie pour donner $b$. La partie médiane $a^{1}$ a complètement disparu et $b$ couvre environ le quart de la surface de la mâchoire. $b$ n'est séparé de $c$ que par une fente très étroite. Il se soude à ce sclérite périphérique d'une part au niveau de $c^{2}$, d'autre part en un point situé au 1/3 distal de $c$.

Les pièces $c$ et $d$ sont soudées sur la ligne médiane du côté distal (fig. 5). L'articulation des sclérites des deux mâchoires se fait comme chez Diclido- 
phora. $i$ vient se placer dans une encôche de $c$ au niveau de la courbure, c'est-à-dire de $c^{2}$.

$k$ vient de même s'articuler sur $d$ au niveau de $d^{2}$.

L'élargissement proximal de $f$ vient s'appuyer sur $a^{2}, a^{3}$ (fig. 4).

Comme chez Diclidophora, un muscle extrinsèque vient aboutir au centre de la fenêtre formée par $a^{3}, c^{3}, c^{2}$ et $b$.

A la partie postérieure du hapteur, entre la première paire de pinces, on distingue parfois une petite languette sur laquelle on trouve deux crochets en fléau de $28 \mu$ de longueur (fig. 1).

A la partie antérieure du corps, la bouche donne accès à une grande cavité buccale où s'ouvrent au centre le pharynx et de chaque côté une ventouse de 80 à $95 \mu$ de long et $45 \mu$ de large. Le pharynx médian est un barillet musculeux de 65 à $130 \mu$ de long et 60 à $100 \mu$ de large. Le tube digestif bifurqué en arrière du pharynx forme de nombreux cæcums latéraux. Les deux branches se réunissent dans la partie postérieure du corps et pénètrent dans la région médiane du hapteur.

Les testicules, dont le nombre varie de 23 à 28 , ont une taille très irrégulière. Ils sont situés dans la moitié postérieure du corps, en avant du hapteur. Le canal déférent large, très contourné, est bourré de spermatozoïdes chez les individus que nous avons examinés. Il remonte en suivant la ligne médiane jusqu'à l'atrium génital situé juste en arrière du pharynx. Avant d'y pénétrer, il se rétrécit et forme un canalicule très étroit, qui débouche dans une large vésicule séminale. Un court conduit réunit cette vésicule à l'ouverture génitale mâle (fig. 3).

L'atrium génital est marqué par un cercle de six à huit épines à pointe dirigée vers le centre. Ces épines, semblables à celles qui existent chez les espèces du genre Diclidophora, ont leur pointe excavée en V (fig. 3).

L'ovaire, situé en avant des testicules, débute sur le côté droit du corps. Il forme une anse sur le côté gauche, revient parallèle à lui-même sur le côté droit où il débouche dans l'oviducte. De cet oviducte cilié, d'abord étroit et contourné, se détache un long canal génito-intestinal à bords extrêmement plissés. Ce canal débouche dans la branche droite du tube digestif. L'oviducte descend alors et, dans l'espace compris entre l'ovaire et les testicules, reçoit le vitelloducte impair, forme une anse postérieure, remonte, se rétrécit énormément dans la portion où il est entouré par les glandes de Mehlis (fig. 2).

L'utérus qui lui fait suite, d'abord élargi à bords plissés (ootype), remonte ensuite en ligne droite jusqu'à l'atrium génital.

Les glandes vitellogènes latérales sont excessivement développées. Postérieurement, les deux bandes latérales se rejoignent en arrière des testicules et pénètrent dans la région médiane du hapteur. Antérieurement, elles semblent se réunir sur la ligne médiane entre l'atrium génital et l'ovaire (fig. 1). Le vitelloducte impair, assez long, débouche dans l'oviducte. 
Il n'y a pas de vagin et nous n'avons pas observé de réceptacle séminal.

Les individus étudiés n'ont pas pondu et ne possédaient pas d'œufs dans leur utérus.

\section{Discussion}

Ce Monogène se classe parmi les Diclidophoridae par le nombre et la structure des pinces et par la disposition de l'appareil génital.

Mais cette famille a été divisée par Sproston en deux sous-familles:

- Diclidophorinae, où les pinces du hapteur fonctionnent comme de véritables pinces. G. Diclidophora Diesing 1850.

- Choricotylinae, où les pinces du hapteur sont aplaties en cupules et fonctionnent comme des ventouses. G. Cyclobothrium Cerfontaine 1895 ; Cyclocotyla Otto 1823 ; Diclidophoropsis Gallien 1937 ; Echinopelma Raecke 1945 ; Heterobothrium Cerfontaine 1895 ; Pedocotyle Mac Callum 1923.

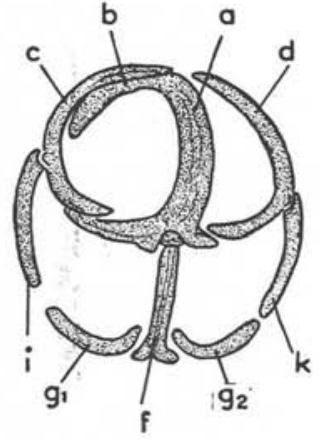

7

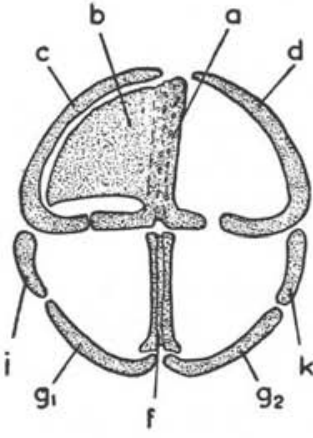

8

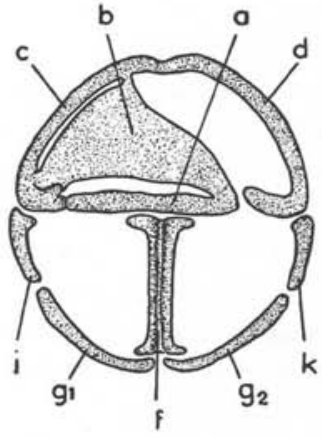

9

Comparaison de la disposition des sclérites chez divers Diclidophoridae

Fig. 7. - Cyclocotyla (Choricotylinae)

Fig. 8. - Diclidophora (Diclidophorinae)

Fig. 9. - Flexophora

(Les pinces sont supposées ouvertes dans les fig. 8 et 9. Lettres dans le texte)

L'espèce que nous avons récoltée, où les pinces fonctionnent comme des pinces, se place dans la sous-famille des Diclidophorinae.

Mais les pièces sclérifiées telles que nous venons de les décrire sont différentes des sclérites qui soutiennent les pinces de Diclidophora. Or, d'après Llewellyn, les pinces de Diclidophora ont une structure particulière que l'on retrouve chez toutes les espèces du genre. 
Ne pouvant classer le parasite d'Ophidium barbatum (L.) dans le genre Diclidophora, nous en ferons une espèce nouvelle, type d'un genre nouveau, pour lesquels nous proposons les noms de Flexophora ophidii n. g., n. sp.

\section{BIBLIOGRAPHIE}

Bychowsky (B. F.), 1957. - Monographie des Monogènes, systématique et phylogénie. Publ. Lab. Zool. Acad. Sc. U.R.S.S., Leningrad, 509 p., 315 fig. (en russe).

Hargis (W. J. Jr), 1955. - Monogenetic Trematodes of Gulf of Mexico fishes. Part. IX. The family Diclidophoridae Fuhrmann 1928. Trans. Amer. Micr. Soc., 74 (4) : 377-388, 19 fig.

Llewellyn (J.), 1958. - The adhesive mechanims of Monogenetic Trematodes : the attachement of species of the Diclidophoridae to the gills of gadoid fishes. J. Mar. Biol. Ass., UK, $37: 67-79,3$ fig., 1 pl.

Robinson (E. S.), 1961. - Some Monogenetic Trematodes from marine fishes of the Pacific. Trans. Amer. Micr. Soc. 80 (3) : 235-266, 61 fig.

Sproston (N. G.), 1946. - A synopsis of the monogenetic Trematodes. Trans. Zool. Soc. London, 25: 185-600, 118 fig.

[Institut de Parasitologie de la Faculté Vétérinaire de Lublin (Pologne)

Station biologique de Sète] 